\title{
Using Automated Analysis of Temporal-Aware SLAs in Logistics ${ }^{\star}$
}

\author{
Carlos Müller, Manuel Resinas, Antonio Ruiz-Cortés \\ Dpto. Lenguajes y Sistemas Informáticos \\ ETS. Ingeniería Informática - Universidad de Sevilla (Spain - España) \\ 41012 Sevilla (Spain - España) \\ \{cmuller, resinas, aruiz\}@us.es
}

\begin{abstract}
Service level agreements (SLAs) establish the terms in which a logistics service may be provided or consumed. During the last years we have been studying techniques to perform an automated analysis of expressive and realistic SLAs, which makes the agreement creation process easier for involved parties. Firstly, we extended WS-Agreement specification to allow to apply any type of validity periods to SLA terms. Later, we dealt with the automated analysis of SLAs by proposing the explaining of SLAs inconsistencies and non-compliance scenarios. In this paper we show how these contributions are necessary to enable a logistic scenario of package tracking by providing examples for each proposal. We also include a final discussion on the convenience of performing a merge of all contributions to enable a better application of SLAs to logistic scenarios.
\end{abstract}

\section{Introduction}

Service-Oriented Computing with its existing set of standards, promotes adaptive supply chain management concepts, flexible and re-configurable logistics service provisioning in supply chains. Thus, service level agreements (SLAs) establish the terms in which a logistics service may be provided or consumed. During the last years we have been studying techniques to perform an automated analysis of expressive and realistic SLAs which makes the agreement creation process easier for involved parties $[3,5,4]$. In order to apply our theories, we used WS-Agreement specification [1], which defines an XML-based language and a protocol for advertising the capabilities and preferences of services providers in templates, and creating agreements based on them. In ICSOC'07 [3], we proposed a temporal domain specific language (DSL) which increases the temporal-awareness of WS-Agreement specification. Such temporal DSL, allows expressive periodical/non-periodical and disjoint/non-disjoint

\footnotetext{
* This work has been partially supported by the European Commission (FEDER), Spanish Government under the CICYT projects Web-Factories (TIN2006-00472), and SETI (TIN2009-07366); and project P07-TIC-2533 funded by the Andalusian local Government.
} 
validity periods to the terms of SLAs. Later in ICSOC'08 [5] and ICSOC'09 [4], we dealt with the automated analysis of SLAs proposing the explaining of SLAs inconsistency and non-compliance scenarios respectively. We also provide a constraint-based solution model and two proof-of-concepts, available for testing at http://www.isa.us.es/wsag. Mentioned [5,4] papers were inspired by previous papers $[2,6]$, which focus on checking whether an SLA is compliant with another one but without providing any explanation for the non-compliance, if any.

Contribution: This paper is focused on applying our previous contributions on automated analysis of SLAs in logistic area. To this end, we take our previous work in $[3,5,4]$ separately, and we apply each one into a package tracking scenario to validate our contributions in logistic area. Furthermore, we provide a final discussion on the convenience of performing a merge of all contributions to a better application to logistic area.

The remainder of the paper is organized as follows: Section 2 describes the used subset of WS-Agreement; Section 3 includes the agreement creation process in a motivating scenario of package tracking providing; Section 4 apply to logistics our contributions of: temporal-aware SLAs in Section 4.1, explaining SLA inconsistencies in Section 4.2, and explaining the non-compliance between templates and agreement offers in Section 4.3; and finally Section 5 conclude this paper and raises a discussion on future work.

\section{WS-Agreement* in a nutshell}

Due to the flexibility and extensibility of WS-Agreement, in $[5,4]$ we focused on WS-Agreement*, which is a subset of WS-Agreement (cf. http://www . isa. us.es/wsag, for details about these differences). WS-Agreement* just imposes several restrictions on some elements of WS-Agreement but it keeps the same syntax and semantics, therefore any WS-Agreement document that follows these restrictions is a WS-Agreement* document. Furthermore, note that, although WS-Agreement* is not as expressive as WS-Agreement, it does allow to express complex agreement documents as those in Figure 1, in which the elements of several WS-Agreement* documents in a packing tracking services providing scenario are depicted.

- Name \& Context identifies the agreement and other information such as a template name and identifier, if any, referring to the specific name and version of the template from which the current agreement is created. For instance, context of Figure 1(c) refers to Template of Figure 1(a).

- Terms can be composed using the three term compositors described in [1]: All $(\wedge)$, ExactlyOne $(\oplus)$, and OneOrMore $(\vee)$. All terms in the document must be included into a main All term compositor. Figure 1(a) includes All and ExactlyOne term compositors. Terms can be divided into:

Service Terms including:

- Service properties must define all variables that are used in the guarantee terms and other agreement elements, explained later. In Figure 
1(a), the variables are the availability of the computing service (Availability), the response time for a request from server, without considering network traffic delays (ResponseTime), and the initial cost for the service (InitCost). The type and general range of values for each variable is provided in an external document such as the ad-hoc XML document depicted in Figure 1(b).

- Service description terms provide a functional description of a service, i.e. the information necessary to provide the service to the consumer. They may set values to variables defined in the service properties (e.g. InitCost $=20$ in Figure 1(a)) or they may set values to new variables. Type and domains are defined in external files such as XML Schemes (e.g. Carrier $=$ MyCarrier in Figure 1(a)).

Guarantee terms describe the service level objectives (SLO) that a specific obligated party must fulfill, and a qualifying condition that specifies the validity condition under which the SLO is applied. For instance the LowerAvailability guarantee term included in Figure 1(a).

A WS-Agreement template is an agreement document with the structure of a WS-Agreement document as described in previous section, but including agreement creation constraints that should be taken into account during the agreement creation process [1]. These Creation Constraints describe the variability allowed by the party that makes the template public. They include (1) Constraints involving the values of one or more terms, for instance the FinalCost definition of "Constraint 1" of Figure 1(a); or (2) Items specifying that a particular variable of the agreement must be present in the agreement offer, typically as a service description term, and its range of values. For instance, the item elements of Figure 1(a) define three variables: the number of dedicated GPS satellites used for locating packages (GPSs), the increase of the cost due to the selected ResponseTime (ExtraRespTimeCost), and the final cost for the service (FinalCost).

\section{Creation of SLAs in a Motivating Logistics Scenario}

A typical interaction process to create agreements using templates and offers, applied to a package tracking service providing scenario, could be as follows: (1) an initiator party, which needs a package tracking service, takes the public template depicted in Figure 1(a) from a responder party. This template describes the agreement terms and some variability that must be taken into account by the initiator in order to achieve an agreement; (2) the initiator creates an agreement offer based on the public template, as Figures 1(c) and 1(d) depicts, and sends it to the responder party; (3) finally, the responder party may accept or not the agreement offer received.

However, an agreement signed by all interested parties should be carefully created because a failure to specify their terms could carry penalties to the initiating or responding party. Therefore, agreement terms of templates and 
agreement offers should be specified in a consistent way, avoiding contradictions between them. In case of inconsistent documents an explanation of why it is inconsistent would be very appealing. For instance, the agreement offer of Figure $1(c)$ includes inconsistent terms emphasized with a cross. Moreover, once established that the agreement offer and the initial template are consistent, parties must ensure the compliance between agreement templates and offers. If they are not compliant, an explanation would make it easier to solve problems between parties. Figure 1(d) depicts a non-compliant agreement offer with template of Figure 1(a), because of the underlined term.

Furthermore, SLAs of logistic services usually require a high degree of temporalawareness. For instance, the package tracking service scenario of Figure 1 could be included in a supply chain with more tasks such as the SMS sending to the final user with package tracking information. If we know that in Christmas period the tracking information is highly demanded by people who want to know whether their packages will arrive on time or not, we need control when and how the service can be requested to satisfy our users. Therefore, we need to include validity periods applied to the whole SLA and to concrete SLA terms.

\section{Our Contributions}

\subsection{Applying Temporal-Aware SLAs in Logistics}

Figure 2 depicts the package tracking service providing scenario of Figure 1 but including the periodical and disjoint validity periods: "Global Period", "LessRespTime Period" and "MoreRespTime Period", depicted in Figure 2(c). "Global Period" informs about when the service can be requested -it is active on 2010 and it just stops the last hour of every Sunday due to maintenance operations-; while "LessRespTime Period" and "MoreRespTime Period" inform about how service can be requested depending on the available number of dedicated servers and GPS satellites used to locate the package: On Monday-Friday from 8:00 to 18:00 the ResponseTime decreases due to a higher number of dedicated servers and GPS satellites used to locate the package at work hours; and the ResponseTime increases at home hours because of a lower number of GPS satellites. In the example we have removed the XML definitions for the validity periods for simplicity (cf. [3], for details on XML validity periods definitions). Figure 2(b) depicts a compliant agreement offer for template of Figure 2(a). In the agreement offer, it is included two optional groups of terms with different values for ResponseTime and GPSs service properties, depending on when the service is requested.

The study of temporal aspects in web services specifications starts with Octavio Martín Díaz's PhD and in ICSOC'05 [2], we overcome the problem of the temporal covering, which involves covering a temporal-aware service demand with several temporal-aware service offers when none of the offers covers totally the demanded period. Later, in ICSOC'07 [3], we present our graphical representation for temporality and we develop a temporal DSL to specify any type of validity periods: (1) periodical/non-periodical, repeated or not on time with a 


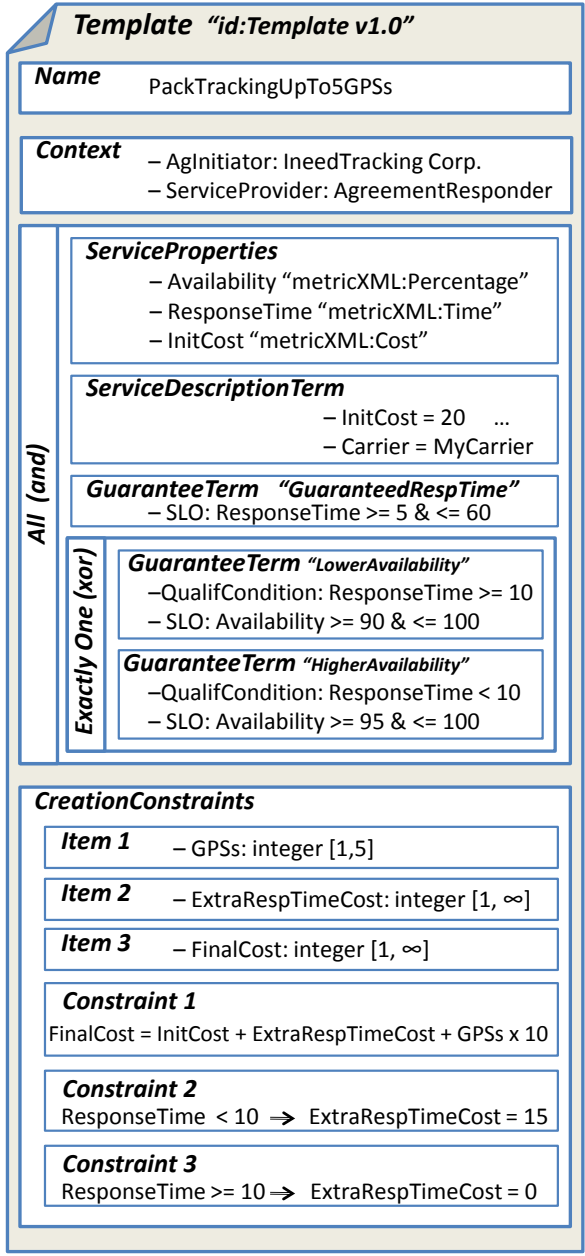

(a) A WS-Agreement template with general and item constraints.

\section{MetricXML}

- Percentage: integer $[1,100]$

- Time: integer $[1, \infty]$

- Cost: integer $[1, \infty]$

(b) Content of the ad-hoc XML document for the variable domains of Figures "a", "c", and "d".

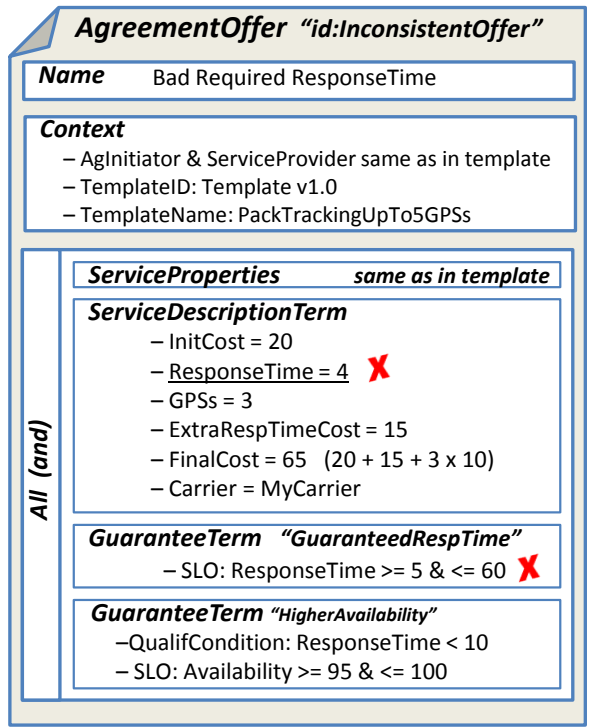

(c) An offer inconsistent with itself.

\section{AgreementOffer "id:Non-CompliantOffer"}

\begin{tabular}{|l|}
\hline Name More GPSs Demanded \\
\hline Context
\end{tabular}

- AgInitiator \& ServiceProvider same as in template

- TemplateID: Template v1.0

- TemplateName: PackTrackingUpTo5GPSs

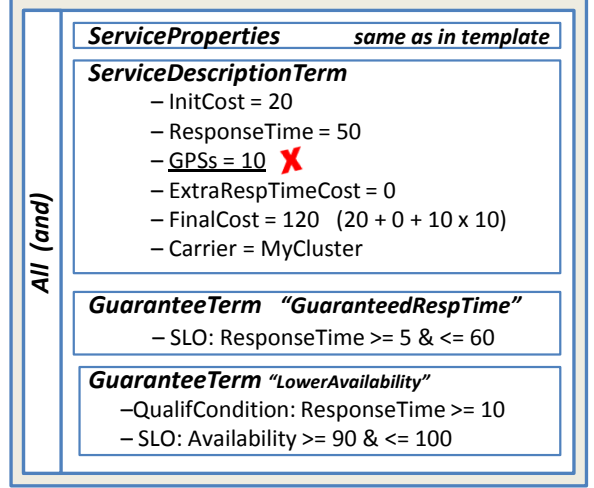

(d) An offer non-compliant with template "a", demanding more dedicated GPS satellites.

Fig. 1. Template and Offers WS-Agreement* documents. 


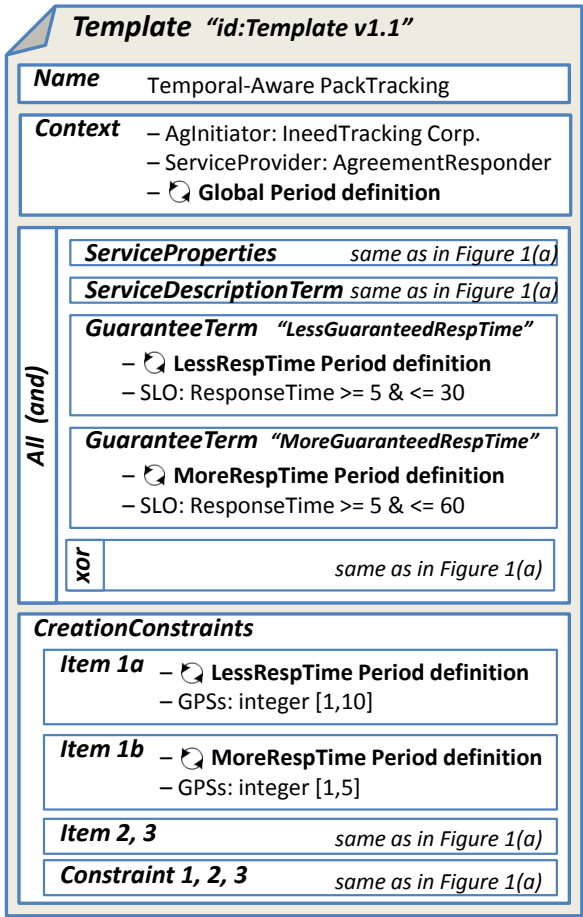

(a) A WS-Agreement template with validity periods for the whole SLA, GuaranteeTerms, and Items.

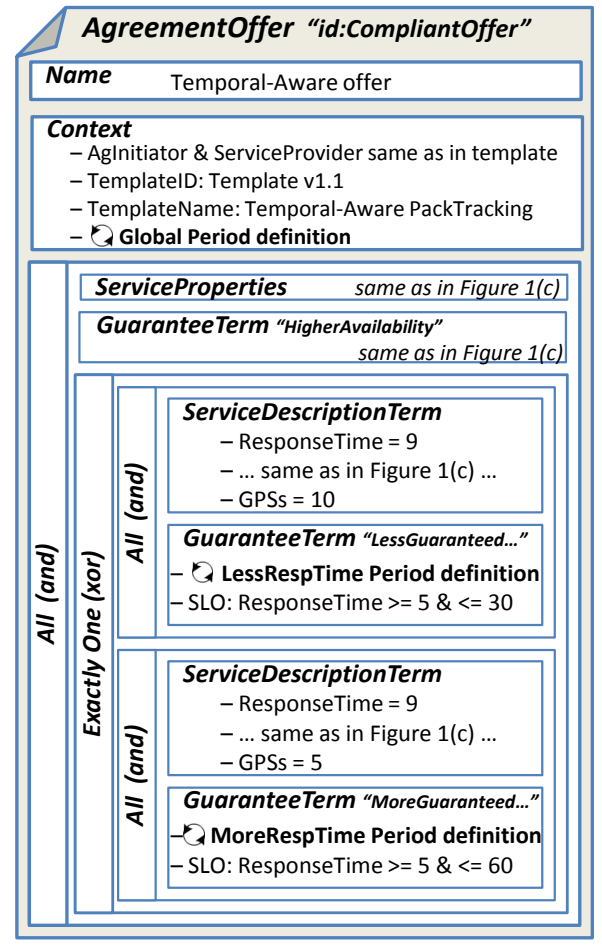

(b) A Compliant offer with template "a", considering the higher value for GPSs and ResponseTime at each validity period.

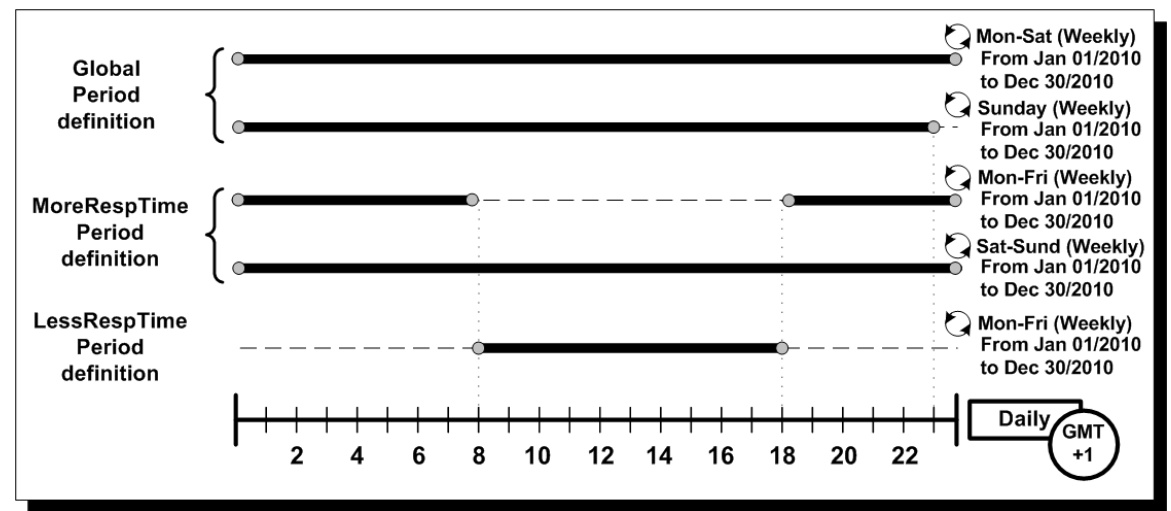

(c) Validity periods definitions for the whole SLA, GuaranteeTerms and Items of Figures "a", and "b".

Fig. 2. Temporal-Aware Template and Offer WS-Agreement* documents. 
concrete frequency, and (2) disjoint/non-disjoint, with gaps inside the period or not. Such temporal DSL is defined as an XML schema which can be downloaded from http: //www.isa.us.es/wsag, but represented in [3] as an UML diagram. We also discuss in [3] how to apply such temporal DSL to the whole agreement or to single terms of WS-Agreement documents.

\subsection{Applying the Explanation of SLA Inconsistencies in Logistics}

The first of our studied main operations of automated analysis of SLAs is "the explaining of SLA inconsistences" and such analysis operation would be of great help for the management of supply chains with logistic services regulated by means of SLAs because a failure to specify their terms could carry penalties to the initiating or responding party. Therefore, agreement terms should be specified in a consistent way, avoiding contradictions between them. However, depending on the complexity of the agreement, this may become a challenging task. The application of our contribution in [5] would obtain error-free SLAs specifications in logistics scenarios. For instance, Figure 1(c) depicts an agreement offer for template of Figure 1(a). However, the agreement offer is not consistent due to a bad value assignment to the ResponseTime property. Thus, if the provider misses this mistake and sign the agreement, the consumer could claim for a compensation and vice versa.

The study of this first operation of automated analysis of SLAs started in [5] where we present a rigorous mapping from WS-Agreement* subset of WSAgreement to constraint satisfaction problems with the objective of analyze such resulting problem and give explanations for inconsistencies, if any. A proof-ofconcept is developed and available for testing at http://www.isa.us.es/wsag.

\subsection{Applying the Explanation of Non-Compliance between Templates and Agreement Offers in Logistics}

The second of our operations of automated analysis of SLAs is "the explaining of non-compliance between several SLAs" and such analysis operation would be of great help for the management of supply chains with logistic services regulated by means of SLAs because it allows to identify why a template and an agreement offer are not compliant. This information can be used to provide solutions by means of checking the reported errors or by means of a negotiation process. In the logistics scenario of Figure 1, a supply chain manager could be interested in the package tracking service described in template 1(a), but during the agreement offer specification depicted in Figure 1(d) he may commit a mistake in the GPSs value assignment. The error is to assign 10 to GPSs, without considering the template creation constraint "Item 1", in which 5 is the higher value allowed for GPSs. In other cases a negotiation process could be needed if the provider is interested in a non-totally-compliant agreement offer.

The study of this second operation of automated analysis of SLAs started in [4] where we take the mechanism of templates and agreement offers of WSAgreement as reference, we include some rigorous definitions of compliance be- 
tween templates and agreement offers, and the explaining of non-compliance scenarios. A proof-of-concept based on constraint satisfaction problems has been developed and it is available for testing at http://www.isa.us.es/wsag.

\section{Conclusions and Discussion on Future Work}

In this paper we have motivated the need for our recent contributions $[3,5,4]$ in logistics scenarios and we have proof that they are applicable by means of examples for each contribution in a package tracking service proving scenario. More specifically, we use the mechanism of templates and agreement offers of WS-Agreement specification to present: (1) a temporal-aware scenario in Figure 2 with any kind of validity periods applied to the whole SLA or to different SLA terms; and (2) an inconsistent and non-compliant scenario in Figure 1 depicting an inconsistent agreement offer and a non-compliant agreement offer with an initial template.

However, such proof has been performed for each contribution on its own. Therefore, we raise now the following question: Is it necessary a merge of contributions to a better application to logistics?

It is obvious that performing a merge of the mentioned contributions we would obtain an automated analysis of SLAs with a high degree of temporalawareness which is very appealing in logistics scenarios because it allows the explaining of inconsistent and non-compliant SLAs with a high degree of temporalawareness. Thus, explaining inconsistent or non-compliant temporal-aware scenarios we could: (1) solve contradictory terms with overlapped validity periods, as for instance "ResponseTime $>=20$ " from Monday to Friday and "ResponseTime $<20$ " from Friday to Sunday; and (2) allow contradictory terms with nonoverlapped validity periods, as for instance 'ResponseTime $>=20$ " from Monday to Friday and "ResponseTime $<20$ " from Saturday to Sunday.

However, such merge is an easy to enunciate problem with a hard solution because we use constraint-based problems as paradigm to solve the explaining of SLAs errors. The inclusion of periodical and disjoint validity periods in such constraint-based problem may obtain a problem too complex to be solved. A possible solution could be to perform a pre-processing of the validity periods which is still under study. Therefore, at the moment we have focused on the study of main operations of automated analysis of SLAs without considering the complex temporal-awareness studied in [3]. In addition, we also have more analysis operations to study, as for instance the analysis of overlapping or differences in an SLA or several SLAs.

\section{References}

1. Andrieux et al. of the OGF Grid Resource Allocation Agreement Protocol WG. Web Services Agreement Specification (WS-Agreement) (v. gfd.107), 2007.

2. O. Martín-Díaz, A. Ruiz-Cortés, A. Durán, and C. Müller. An approach to temporalaware procurement of web services. In 3rd ICSOC, pages 170-184, 2005. 
3. C. Müller, O. Martín-Díaz, A. Ruiz-Cortés, M. Resinas, and P. Fernández. Improving Temporal-Awareness of WS-Agreement. In Proc. of the $5^{\text {th }}$ ICSOC, pages 193-206. Springer Verlag, 2007.

4. C. Müller, M. Resinas, and A. Ruiz-Cortés. Explaining the Non-Compliance between Templates and Agreement Offers in WS-Agreement*. In Proc. of the rth ICSOC, Sweden, Stockholm, 2009. Springer Verlag.

5. C. Müller, A. Ruiz-Cortés, and M. Resinas. An Initial Approach to Explaining SLA Inconsistencies. In Proc. of the $6^{\text {th }}$ ICSOC. Springer Verlag, 2008.

6. A. Ruiz-Cortés, O. Martín-Díaz, A. Durán, and M. Toro. Improving the Automatic Procurement of Web Services using Constraint Programming. Int. Journal on Cooperative Information Systems, 14(4), 2005. 